\title{
Search for A-F Spectral type pulsating components in Algol-type eclipsing binary systems ${ }^{\star}$
}

\author{
S.-L. Kim ${ }^{1}$, J. W. Lee ${ }^{2}$, S.-G. Kwon ${ }^{1}$, J.-H. Youn ${ }^{1}$, D. E. Mkrtichian ${ }^{3,4}$, and C. Kim ${ }^{5}$ \\ 1 Korea Astronomy Observatory, Daejeon, 305-348, Korea \\ 2 Department of Astronomy and Space Science, Chungbuk National University, Cheongju, 361-763, Korea \\ 3 Astronomical Observatory, Odessa National University, Shevchenko Park, Odessa, 65014, Ukraine \\ ${ }^{4}$ Astrophysical Research Center for the Structure and Evolution of the Cosmos, Sejong University, Seoul, 143-747, Korea \\ 5 Department of Earth Science Education, Chonbuk National University, Chonju, 561-756, Korea
}

Received 24 February 2003 / Accepted 2 April 2003

\begin{abstract}
We present the results of a systematic search for pulsating components in Algol-type eclipsing binary systems. A total number of 14 eclipsing binaries with A-F spectral type primary components were observed for 22 nights. We confirmed small-amplitude oscillating features of a recently detected pulsator TW Dra, which has a pulsating period of 0.053 day and a semi-amplitude of about $5 \mathrm{mmag}$ in $B$-passband. We discovered new pulsating components in two eclipsing binaries of RX Hya and $\mathrm{AB}$ Per. The primary component of RX Hya is pulsating with a dominant period of 0.052 day and a semi-amplitude of about 7 mmag. AB Per has also a pulsating component with a period of 0.196 day and a semi-amplitude of about $10 \mathrm{mmag}$ in $B$-passband. We suggest that these two new pulsators are members of the newly introduced group of mass-accreting pulsating stars in semi-detached Algol-type eclipsing binary systems.
\end{abstract}

Key words. stars: binaries: eclipsing - stars: variables: $\delta$ Sct - stars: individual: TW Dra, RX Hya, AB Per

\section{Introduction}

$\delta$ Scuti-type pulsating stars are located at the classical instability strip on and above the main sequence with spectral types from A to early $\mathrm{F}$. These variables have short pulsation periods of less than 0.3 days and half of them have visual amplitudes smaller than 0.05 . A total of $636 \delta$ Scuti stars are listed in a recent catalogue by Rodríguez et al. (2000).

$\delta$ Scuti-type pulsating components in eclipsing binary systems are attractive observing targets for asteroseismology because we can identify their non-radial modes using the amplitude and phase changes during the eclipse. The masses and radii for each component in eclipsing binaries could be accurately determined, so that it should help to model the pulsation spectra. Nevertheless, probably due to difficulty in observing small-amplitude oscillation in comparison with large light variation caused by the eclipsing phenomenon, only nine $\delta$ Scutitype pulsating components in eclipsing binary systems were reported in a general review on $\delta$ Scuti stars (Rodríguez \& Breger 2001). In addition, two new variables were recently discovered: TW Dra (Kusakin et al. 2001) and GK Dra (Dallaporta et al. 2002). Among these eleven variables, three stars of AB Cas

Send offprint requests to: S.-L. Kim, e-mail: slkim@kao.re.kr

* Table 4 is only available in electronic form at the CDS via anonymous ftp to cdsarc.u-strasbg.fr $(130.79 .128 .5)$ or via http://cdsweb.u-strasbg.fr/cgi-bin/qcat?J/A+A/405/231
(Rodríguez et al.1998), RZ Cas (Ohshima et al. 2001) and Y Cam (Kim et al. 2002a) have been studied photometrically in detail at a single observing site. From the multisite photometric campaign of AS Eri, Mkrtichian et al. (2003a) detected three oscillation modes around $24 \mathrm{~min}$. Including the $22 \mathrm{~min}$ oscillation in RZ Cas, these are the shortest periods excited in non-magnetic A-F spectral type main-sequence stars. Shortperiod oscillations for the Algol-type eclipsing binary AI Dra were also reported by Narusawa et al. (2002) but were not confirmed later (Kiss 2002).

Mkrtichian et al. (2003a) suggested a new pulsating group defined as "the (B)A-F spectral type mass-accreting mainsequence pulsating stars in semi-detached Algol-type binary systems". Their pulsation characteristics are similar to those of normal $\delta$ Scuti-type stars, but their evolution scenario is very different due to mass accretion. The authors proposed the oEA (oscillating EA) designation for this group of pulsators to separate them from the General Catalogue of Variable Stars (GCVS) designation of EA/DSCT which includes also detached binary systems with normal $\delta$ Scuti-type pulsating components. Among the eleven stars mentioned, six variables belong to this group including the well-observed four stars discussed as well as the recently discovered pulsator TW Dra.

In collaboration with the Central Asian Network (CAN) group (Mkrtichian et al. 2002), we have been performing a photometric survey project to search for $\delta$ Scuti-type pulsating 
Table 1. Observing log.

\begin{tabular}{|c|c|c|c|c|c|}
\hline Stars & Date & Run & Filt. & Seeing & Note \\
\hline \multirow[t]{2}{*}{ TW Dra } & May 1,2002 & 8.64 & $B$ & 2.6 & - \\
\hline & May 23, 2002 & 7.44 & $B$ & $2 . .3$ & - \\
\hline \multirow[t]{2}{*}{ RX Hya } & Feb. 28, 2002 & $2 \mathrm{~h} 26$ & $B$ & $3{ }^{\prime \prime} 0$ & - \\
\hline & Mar. 1, 2002 & 4.76 & $B$ & $2 . .5$ & - \\
\hline \multirow[t]{5}{*}{ AB Per } & Dec. 23, 2001 & 9.21 & V & 2.7 & - \\
\hline & Jan. 31, 2002 & 5.42 & $B$ & $2 . .8$ & - \\
\hline & Sep. 18, 2002 & 6.63 & $B$ & 2.5 & - \\
\hline & Sep. 23, 2002 & 5.27 & $B$ & 2.2 & - \\
\hline & Sep. 24, 2002 & $5^{\mathrm{h}} .72$ & $B$ & 2.0 & - \\
\hline \multirow{3}{*}{ X Tri } & Dec. 8, 2001 & 6.54 & $V$ & 2.3 & Min. I \\
\hline & Dec. 9,2001 & 1.22 & $V$ & $2 . .7$ & - \\
\hline & Dec. 24, 2001 & 4.39 & V & 2.2 & Min. II \\
\hline \multirow[t]{2}{*}{ XZ UMa } & Dec. 28,2001 & 4.40 & V & $2 . .7$ & - \\
\hline & Jan. 30, 2002 & $5^{\mathrm{h}} .14$ & $B$ & 4.0 & - \\
\hline AC UMa & Jan. 31, 2002 & 6.53 & $B$ & 2.5 & - \\
\hline RW Per & Dec. 25,2001 & 8.41 & V & 2.6 & Min. II \\
\hline \multirow[t]{2}{*}{ ST Per } & Feb. 1, 2002 & 4.73 & $B$ & $2{ }^{\prime \prime} 1$ & Min. I \\
\hline & Dec. 14, 2002 & $5^{\mathrm{h}} .02$ & $B$ & $2 . .8$ & - \\
\hline \multirow[t]{2}{*}{ RV Per } & Dec. 27, 2001 & 3.07 & V & $3 .^{\prime \prime} 0$ & - \\
\hline & Feb. 28, 2002 & 3.59 & $B$ & $3{ }^{\prime \prime} 0$ & - \\
\hline \multirow[t]{2}{*}{ CP And } & Dec. 24, 2001 & $2^{\mathrm{h}} .12$ & V & $2 . .4$ & - \\
\hline & Dec. 2, 2002 & 3.29 & $B$ & $2 . .5$ & - \\
\hline VZ Leo & Dec. 30, 2001 & $5^{\mathrm{h}} 64$ & $V$ & $2{ }^{\prime \prime} 6$ & - \\
\hline \multirow[t]{2}{*}{ RS Cep } & Dec. 28, 2001 & 3.65 & V & $2 . .4$ & - \\
\hline & Nov. 30, 2002 & 3.24 & $B$ & 1 .. 8 & - \\
\hline Z Dra & Mar. 24, 2002 & 4. 12 & $B$ & 2.7 & - \\
\hline \multirow[t]{2}{*}{ RW CrB } & Mar. 24, 2002 & $3^{\mathrm{h}} 30$ & $B$ & $2 . .7$ & Min. I \\
\hline & Sep. 18, 2002 & 2.09 & $B$ & $3 . .0$ & - \\
\hline
\end{tabular}

components in eclipsing binary systems from September 2001 onwards at the Sobaeksan Optical Astronomy Observatory (SOAO) in Korea. The main purpose of the project is to increase the number of these interesting objects known and then to enable us to carry out statistical studies on their physical characteristics. We selected about 110 observing targets whose primary components have A-F spectral types. Several pulsating candidates suspected from the Hipparcos satellite data were observed with higher priorities. In this paper, we present the first results of the survey project.

\section{Observation and data reduction}

We carried out CCD photometric observations for 22 nights from December 2001 to December 2002, using a SITe 2K CCD camera attached to the $61 \mathrm{~cm}$ telescope at the SOAO. The CCD chip has $2048 \times 2048$ pixels and a pixel size of $24 \mu \mathrm{m}$. The field of view (FOV) of the CCD image is about $20.5 \times 20.5$ on the $\mathrm{f} / 13.5$ Cassegrain focus of the telescope. In order to detect small-amplitude short-period oscillations, we monitored most of the observing targets for larger than 5 hours with only one filter. We changed the passband from $V$ to $B$ on January 2002 because $\delta$ Scuti-type pulsators show the largest amplitudes in $B$-passband. The detailed observation log is listed in Table 1 .

Using the IRAF/CCDRED package, we processed timeseries CCD frames to correct the bias level and the pixel-to-pixel inhomogeneity of quantum efficiency (flat fielding). A star with similar brightness to the variable star was chosen as a comparison star. A few tens of stars in each observing field were also monitored to check their variabilities; we found three new field $\delta$ Scuti stars in these observing runs (Kim et al. 2002b). Using the IRAF/APPHOT package, we applied simple aperture photometry to get instrumental magnitudes with an aperture radius of 6.' 0 . For the data obtained under poor seeing larger than 3.' 5 and for those of X Tri which has a faint companion $\operatorname{star}(\Delta V=4.3)$ at a distance of about 6. .' 0 , we adopted a large aperture radius of 9.' 0 . Differential magnitudes were calculated from the standard differential photometric method.

In order to remove slow light variations caused by the eclipsing phenomenon, we constructed synthetic eclipsing curves from the Wilson-Devinney (1971) method or low-order (typically, third-order) polynomial fitting curves. Then we performed a Discrete Fourier analysis (Kim \& Lee1996) to detect short-period oscillations from the residuals, the subtraction values of the curves from the data; in this paper, it is also called reduced light variations.

On the basis of the results for a previously known pulsator, TW Dra, we deduced the detection limit of our observing data to be about 4 mmag in semi-amplitude. It corresponds to the power value of $0.04 \times 10^{-4}$ in the power spectra; the square root value of 4.0 (normalization factor) $\times 0.04 \times 10^{-4}$ power is 4 mmag.

\section{Results for individual stars}

Physical parameters and our photometric results for 14 target stars are summarized in Table 2. Orbital phases of these eclipsing binaries, except for AB Per and X Tri (see each subsection), were calculated from light elements in the GCVS (Kholopov et al. 1988).

\subsection{TW Dra}

TW Dra is a semi-detached Algol-type eclipsing binary system with A8V primary and K0III secondary components. It has undergone a rapid secular increase of orbital period, $\mathrm{d} P / \mathrm{d} t=$ $+4.43 \times 10^{-6}$ days/year, which might result from a dynamical mass transfer from the secondary component to the primary one (Qian \& Boonrucksar 2002). Within the framework of the CAN project to search for pulsations in Algols, Kusakin et al. (2001) discovered the first small-amplitude oscillations of the primary component with a dominant frequency of $17.99 \mathrm{c} / \mathrm{d}$ (cycles per day) and a semi-amplitude of about $2.1 \mathrm{mmag}$ in the $V$-passband.

Figure 1 displays reduced light variations and power spectra of TW Dra obtained for two nights. A peak near $7 \mathrm{c} / \mathrm{d}$ in the lower left panel seems to originate from a large bump at the orbital phase around 0.72 . This bump may be caused by gas stream effects in semi-detached Algol-type binary systems and is normally seen shortly after the orbital phase of 0.75 (Walter 1980), which is different from our result. Our orbital phases might not be real values, resulting from the large orbital period variations and the out-of-date GCVS database for TW Dra. We tested the effect of this bump after removing the data before 
Table 2. Physical parameters of the observing targets.

\begin{tabular}{cccccl}
\hline \hline Stars & Max. $^{\dagger}$ & Var. type $^{\dagger}$ & Sp. type $^{\dagger}$ & $P_{\text {orb }}$ (day) $)^{\dagger}$ & Pulsation \\
\hline TW Dra & 8.0 & EA/SD & A8V+K0III & 2.806847 & $P_{\text {pul }}=0.053, \Delta \mathrm{B} \approx 0.010$ \\
RX Hya & 8.9 & EA/SD & A8 & 2.281645 & $P_{\text {pul }}=0.052, \Delta \mathrm{B} \approx 0.014$ \\
AB Per & 10.4 & EA/SD & A5 & 7.1602886 & $P_{\text {pul }}=0.196, \Delta \mathrm{B} \approx 0.020$ \\
X Tri & 8.55 & EA/SD & A5V+G0V & 0.9715352 & weak signals around 30 and 45 c/d ? \\
XZ UMa & 10.1 & EA/SD & A5+F9 & 1.22232 & - \\
AC UMa & 10.3 & EA/SD & A2 & 6.854939 & - \\
RW Per & 9.68 & EA/D & A5IIIe+G0III & 13.198904 & - \\
ST Per & 9.52 & EA/SD & A3V+G & 2.648315 & - \\
RV Per & 10.3 & EA/SD & A2 & 1.9734926 & - \\
CP And & 11.4 & EA/SD & A5 & 3.60894 & - \\
VZ Leo & 11.8 & EA/SD & A5 & 1.089906 & - \\
RS Cep & 10.2 & EA/SD & A5IIIe $+\mathrm{G}$ & 12.420105 & - \\
Z Dra & 10.8 & EA/SD & F4V & 1.357456 & - \\
RW CrB & 10.2 & EA/SD & F2V & 0.7264114 & - \\
\hline
\end{tabular}

${ }^{\dagger}$ From the GCVS (Kholopov et al. 1988).
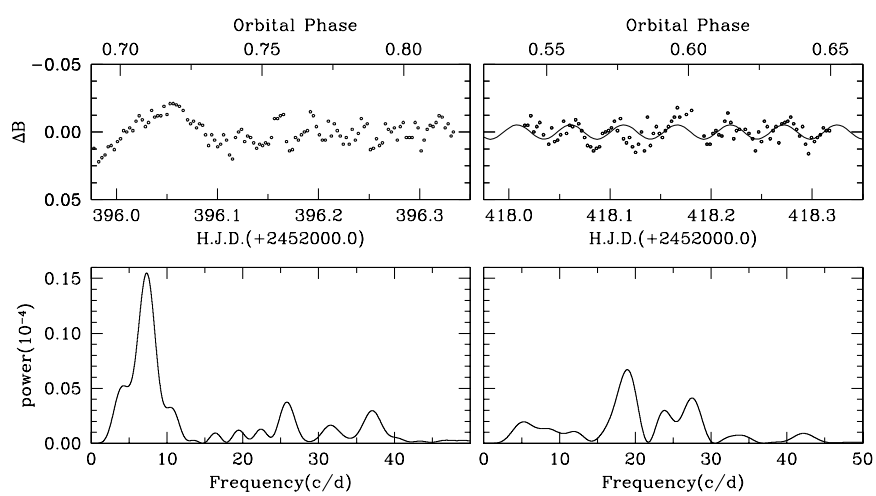

Fig. 1. Reduced light variations (upper) and power spectra (lower) of TW Dra obtained for two nights. A sinusoidal curve with a frequency of $18.95 \mathrm{c} / \mathrm{d}$ and a semi-amplitude of $5.3 \mathrm{mmag}$ is superimposed in the upper right panel.

HJD 2452396.1 but could not find noticeable changes of power spectra in the high frequency region larger than $15 \mathrm{c} / \mathrm{d}$.

Around secondary minimum orbital phases (HJD 2452418.0), we detected an oscillation pattern with a peak frequency of $18.95 \mathrm{c} / \mathrm{d}$ and a semi-amplitude of $5.3 \mathrm{mmag}$ in $B$-passband. In comparison with the previous value of $17.99 \mathrm{c} / \mathrm{d}$, our result appears to be $1 \mathrm{c} / \mathrm{d}$ alias frequency. The secondary peak is shown near $26 \sim 28 \mathrm{c} / \mathrm{d}$ in both nights. We could also find a peak at a similar frequency region in the previous result (in Fig. 3 of Kusakin et al. 2001). Therefore, we suggest that TW Dra is a multi-periodic pulsator with a dominant frequency about $18 \mathrm{c} / \mathrm{d}$ (or $19 \mathrm{c} / \mathrm{d}$ ) and another one around $27 \mathrm{c} / \mathrm{d}$. The ratio of orbital period to pulsating one is about 51 .

\subsection{RX Hya}

RX Hya is a semi-detached Algol-type eclipsing binary system with A or F main-sequence primary and $\mathrm{K}$ subgiant secondary components. Vyas \& Abhyankar (1989) suggested that the secondary is filling its Roche lobe and transferring masses to the
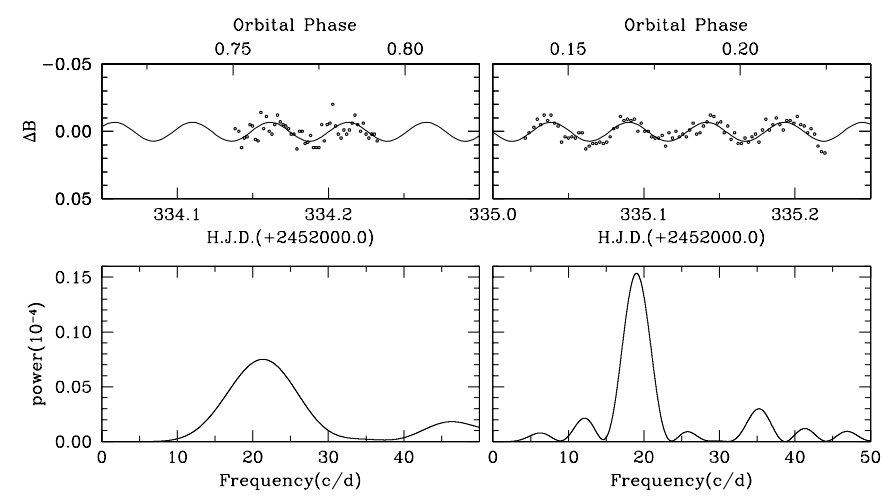

Fig. 2. Reduced light variations (upper) and power spectra (lower) of RX Hya obtained for two nights. Sinusoidal curves with a frequency of $19.39 \mathrm{c} / \mathrm{d}$ and a semi-amplitude of 7.0 mmag are superimposed in the upper panels.

primary with a rate of $3.0 \times 10^{-6} M_{\odot} /$ year, estimated from orbital period changes.

The brightest star BD-07 $2718(V=9 \cdot 70,(B-V)=$ $1^{\mathrm{m}} 40$ ) in the observing field of RX Hya was chosen as a comparison star. Because the color index of the comparison star is too red relative to the variable star and the data were obtained at large airmass ranging from 1.4 to 2.5 , we should correct the second-order atmospheric extinction effect: an airmass-related slow light variation due to the large color difference. Figure 2 displays reduced light variations and power spectra of RX Hya obtained for two nights. We observed the variable star during out-of eclipsing orbital phases around 0.77 (HJD 2 452334.2) and 0.18 (HJD 2452 335.1).

Oscillation features of the variable star RX Hya were clearly detected on both nights. We obtained a dominant frequency of $19.39 \mathrm{c} / \mathrm{d}$ and a semi-amplitude of about $7.0 \mathrm{mmag}$ in $B$-passband. Considering the spectral type, sinusoidal light curves, frequency and amplitude of pulsation, and the membership in a semi-detached Algol-type system, we suggest that the primary component of RX Hya is a new member of the oscillating EA stars. 


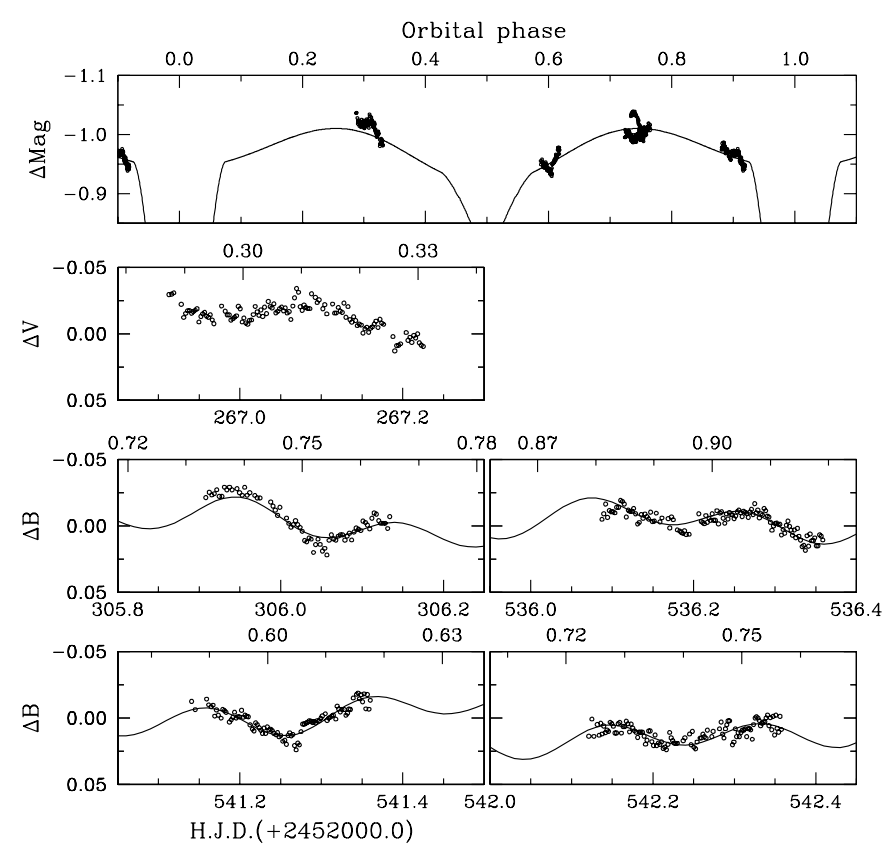

Fig. 3. Light variations of the variable star $A B$ Per. The synthetic light curves represented by solid lines in the top panel were constructed using the Wilson-Devinney (1971) method. The lower five panels show residuals after fitting the curves to the data. Sinusoidal curves obtained from the multiple frequency analysis are superimposed on the residuals in four $B$-band panels.

\section{3. $A B P e r$}

AB Per is a semi-detached Algol-type eclipsing binary system with an orbital period of about 7.16 days. Its primary and secondary components were known to have A and F spectral type (Struve 1945), respectively. The Hipparcos data of AB Per show relatively large scatters in out-of eclipsing phases in comparison with observational errors (ESA 1997), implying the possibility that $\mathrm{AB}$ Per has the other variability sources besides the eclipsing phenomenon: e.g. pulsation of a component in the binary system.

A star, HD 275605, nearby AB Per was chosen as a comparison star. It has similar brightness and color index to the variable star and did not show any peculiar light variation during the observing runs (see Kim et al. 2002b). Because light elements in the GCVS might have some problems (Isles 1991), we calculated the orbital phases from the following elements which were newly derived from all previously-known minimum epochs (Kreiner et al. 2001),

$$
\text { Min HJD }=2422987.3254+7.16007115 \times E .
$$

Light variations of $\mathrm{AB}$ Per are shown in Fig. 3. In order to remove the eclipsing light variations, we constructed synthetic curves using the Wilson-Devinney (1971) method and photometric parameters from the literature (Brancewicz \& Dworak 1980; Budding 1985; van Hamme 1993). Residuals after fitting the curves to the data show short-term variabilities with a period of about 0.2 day and $\Delta B \approx 0 .{ }^{\mathrm{m}} 02$.

We estimated oscillating periods from the multiple frequency analysis (Kim \& Lee 1996), using only the $B$-band data. Figure 4 displays the power spectra of the residuals.
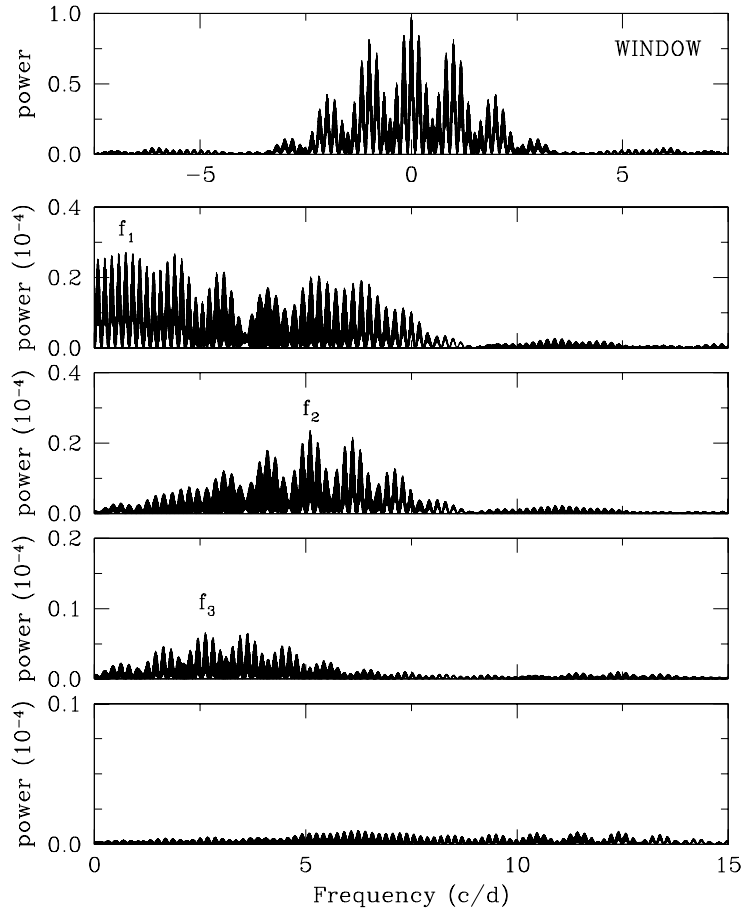

Fig. 4. Power spectra of AB Per. Window spectrum is in the top panel. The successive pre-whitening procedure shows three frequencies of $f_{1}=0.747 \mathrm{c} / \mathrm{d}, f_{2}=5.106 \mathrm{c} / \mathrm{d}$ and $f_{3}=2.624 \mathrm{c} / \mathrm{d}$.

The successive prewhitening procedure shows three frequencies of $f_{1}=0.747 \mathrm{c} / \mathrm{d}, f_{2}=5.106 \mathrm{c} / \mathrm{d}$ and $f_{3}=2.624 \mathrm{c} / \mathrm{d}$. The first frequency was selected in order to remove long-term trends of the residuals. The second frequency was clearly detected in the power spectra. The third frequency with the smallest amplitude is uncertain as to whether it is a real frequency of the star; $f_{3}$ seems to be a sub-harmonic frequency of $f_{2}, f_{3} \sim 1 / 2 f_{2}$. More intensive and long time-based observations of AB Per are needed.

The second period of 0.196 day $(5.106 \mathrm{c} / \mathrm{d})$ is much smaller in comparison with the orbital period of about 7.16 day for AB Per. The variable star AB Per is a member of a semidetached eclipsing binary system whose revolution and rotation are supposed to be synchronized (Glazunova 1999). Therefore, the second frequency $f_{2}$ might not originate from rotationinduced variabilities such as the ellipsoidal configuration or surface inhomogeneity. Instead, it is more reasonable that $f_{2}$ is a pulsating frequency of the primary component. Considering its physical parameters and pulsational characteristics, we suggest that the primary component of AB Per is also a new member of the oscillating EA stars.

\subsection{The other stars}

$\mathrm{X}$ Tri is a semi-detached eclipsing binary with a variable orbital period. Qian (2002) found a secular period decrease of $\mathrm{d} P / \mathrm{d} t=-1.42 \times 10^{-7}$ days $\mathrm{yr}^{-1}$ and also several small-scaled irregular period jumps. He suggested that these period changes may be caused by orbital angular momentum loss via variable magnetic coupling and gravity coupling between two components. We obtained the data of X Tri for three nights, of which 

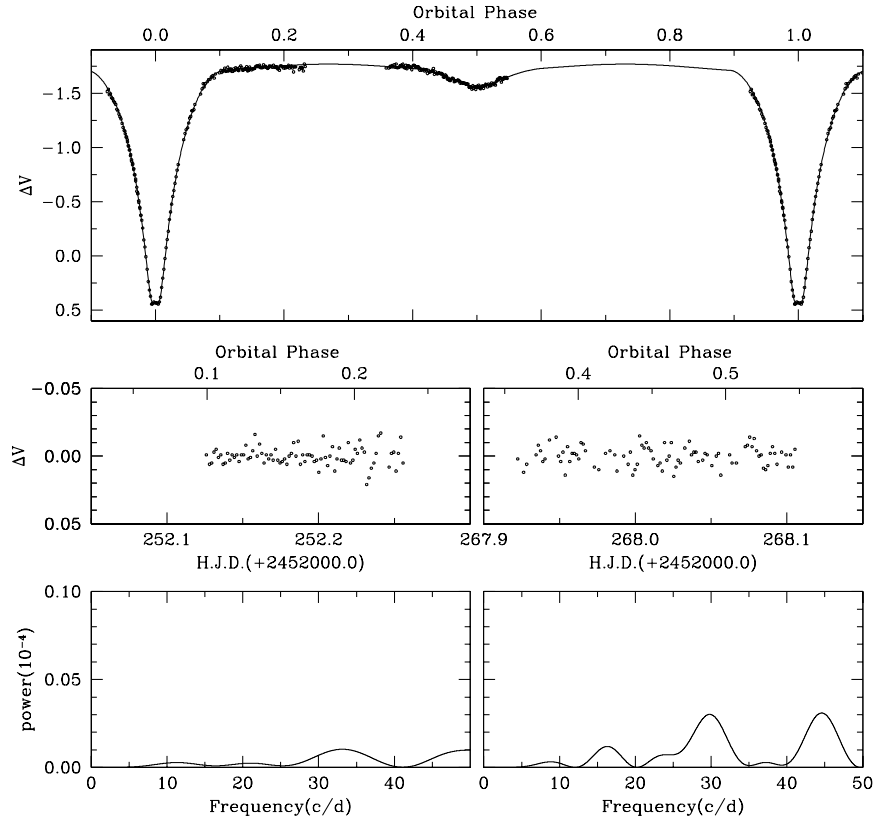

Fig. 5. Eclipsing light curves (top), reduced light variations (middle) and power spectra (bottom) of X Tri. We examined only the data during out-of the primary eclipsing phase in order to detect pulsating features of the primary component.
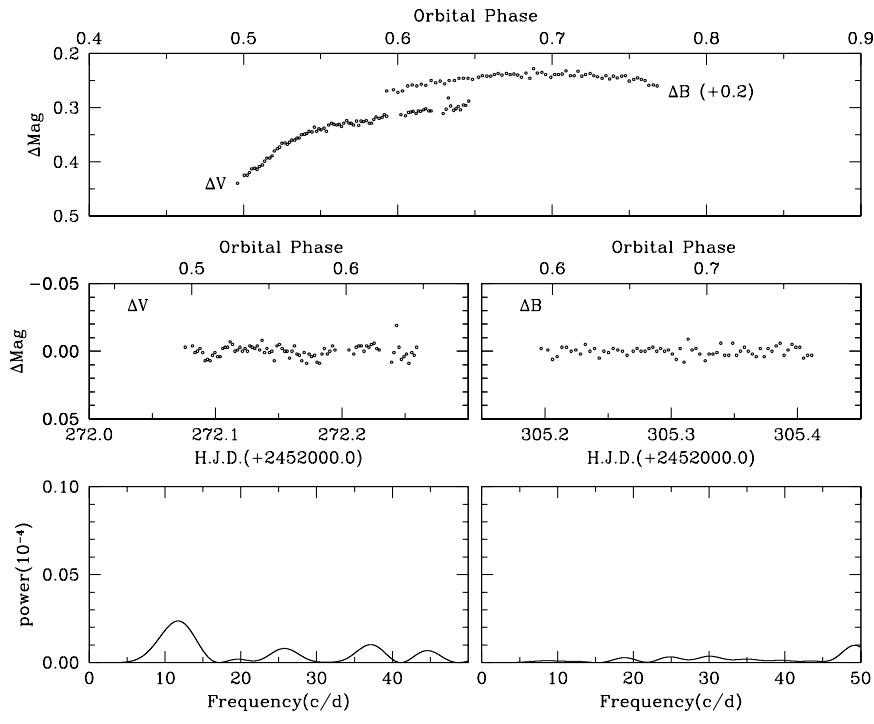

Fig. 6. Eclipsing light curves (top), reduced light variations (middle) and power spectra (bottom) of XZ UMa obtained for two nights.

two nights are around the primary minimum phase. Figure 5 displays light variations and power spectra of $X$ Tri. The orbital phases calculated from light elements in the GCVS were slightly shifted from our primary minimum, probably due to orbital period variations of $\mathrm{X}$ Tri, so that we used a new minimum epoch (see Table 3). In the power spectra, we could not detect any noticeable signal, even if two weak peaks are shown around $30 \mathrm{c} / \mathrm{d}$ and $45 \mathrm{c} / \mathrm{d}$ during the secondary minimum epoch. More precise photometric data with the $B$ filter would be required to confirm these small-amplitude oscillations.

The eclipsing binary XZ UMa was observed for two nights around the secondary minimum phase. Figure 6 displays light
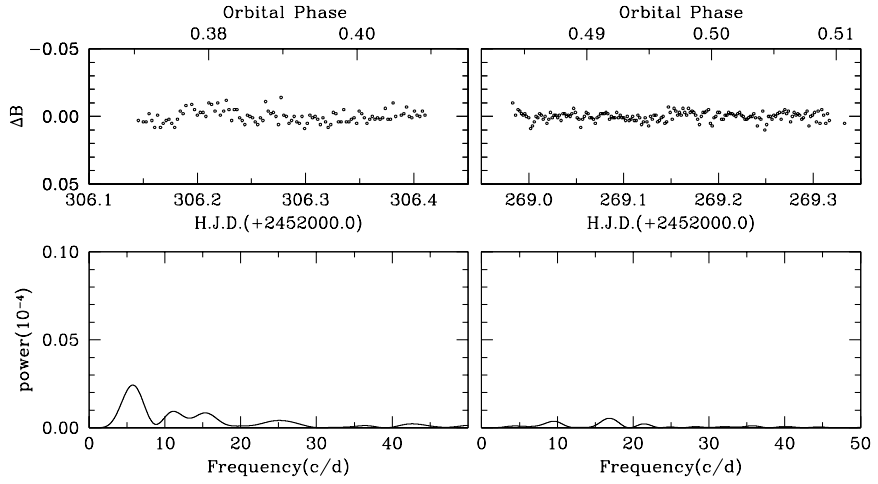

Fig. 7. Reduced light variations (upper) and power spectra (lower) of AC UMa (left) and RW Per (right).

Table 3. Minimum epochs obtained in this study.

\begin{tabular}{cll}
\hline \hline Stars & Minimum epochs (HJD) & Type \\
\hline X Tri & $2452252.02925 \pm 0.00004$ & Min. I \\
X Tri & $2452268.0613 \pm 0.0004$ & Min. II \\
RW CrB & $2452358.3151 \pm 0.0001$ & Min. I \\
ST Per & $2452307.01686 \pm 0.00007$ & Min. I \\
RW Per & $2452269.1231 \pm 0.0002$ & Min. II \\
\hline
\end{tabular}

variations and power spectra of XZ UMa. The $B$-band spectrum in the bottom right panel shows clearly that XZ UMa does not have any short-period oscillation. A small peak near $12 \mathrm{c} / \mathrm{d}$ in the bottom left panel might result from incompletely reduced light variations. It was a little difficult for us to fit the data with a low-order polynomial curve because the data consist of the secondary minimum phase as well as the out-of eclipsing one.

We monitored two eclipsing binaries, AC UMa and RW Per, for more than 6 hours on one night for each object. Their reduced light variations and power spectra are shown in Fig. 7. No short-period oscillating feature was detected for these two binaries. For the other seven stars, ST Per, RV Per, CP And, VZ Leo, RS Cep, Z Dra and RW CrB, we also could not find periodic light variations within the detection limit of about 4 mmag in semi-amplitude.

During the observing run, we obtained some brightness minimum epochs for four eclipsing binaries (Table 3). The epochs were estimated from the well-known method by Kwee $\&$ van Woerden (1956). These data can be used to study longterm variations of their orbital periods.

\section{Conclusion}

We monitored 14 eclipsing binary systems to search for $\delta$ Scutitype pulsating components. The photometric data are listed in Table 4, which is available in electronic form at the CDS. Small-amplitude oscillations for a recently detected pulsator TW Dra were confirmed. We discovered new pulsating components in two semi-detached Algol-type eclipsing binary systems: RX Hya and AB Per. Our results show that the primary components of RX Hya and $\mathrm{AB}$ Per are pulsating with a dominant pulsating period of 0.052 day and of 0.196 day, 
respectively. On the basis of their semi-detached configuration and pulsational characteristics, we identify these two new variables as members of the newly introduced pulsating group, oscillating EA stars (Mkrtichian et al. 2003a). The number of variable stars that belong to this interesting pulsating group has increased eight (Mkrtichian et al. 2003b).

We detected small-amplitude oscillation features for three eclipsing binaries out of fourteen observing targets. It does not rule out the possibility that some of the other binary systems have pulsating components but their oscillating amplitudes are smaller than our detection limit of about 4 mmag in semiamplitude. However, it does not imply that the incidence of $\delta$ Scuti-type pulsating components in Algol-type eclipsing binary systems with an $\mathrm{A}-\mathrm{F}$ spectral type primary component is as high as about $20 \%$. This is because we chose first several pulsating candidates suspected from the Hipparcos satellite data and monitored them with higher priorities.

Acknowledgements. We would like to thank the referee, Dr. Eloy Rodríguez, for valuable comments on the manuscript. We also thank Dr. Yong-Jae Moon for his careful reading and Prof. Chun-Hwey Kim for his help using the $(\mathrm{O}-\mathrm{C})$ database of eclipsing binaries. This research made use of the SIMBAD database, operated at CDS, Strasbourg, France. This work was supported by Korea Research Foundation Grant (KRF-2001-015-DP0612).

\section{References}

Brancewicz, H. K., \& Dworak, T. Z. 1980, Acta Astron., 30, 501 Budding, E. 1985, PASP, 97, 584

Dallaporta, S., Tomov, T., Zwitter, T., \& Munari, U. 2002, Inf. Bull. Variable Stars, No. 5312

ESA, 1997, The Hipparcos and Tycho Catalogues, ESA, SP-1200

Isles, J. E. 1991, J. Br. Astron. Assoc., 101, 219

Glazunova, L. V. 1999, Astron. Lett., 25, 467

Kholopov, P. N., Samus, N. N., Frolov, M. S., et al. 1988, in General Catalogue of Variable Stars, 4th ed. (Moscow: Nauka Publishing House)
Kim, S.-L., \& Lee, S.-W. 1996, A\&A, 310, 831

Kim, S.-L., Lee, J. W., Youn, J.-H., Kwon, S.-G., \& Kim, C. 2002a, A\&A, 391, 213

Kim, S.-L., Kwon, S.-G., Youn, J.-H., Kyeong, J.-M., \& Lee, J. W. 2002b, Inf. Bull. Variable Stars, No. 5244

Kiss, L. L. 2002, Inf. Bull. Variable Stars, No. 5355

Kreiner, J. M., Kim, C.-H., \& Nha, I.-S. 2001, in An Atlas of (O-C) Diagrams of Eclipsing Binary Stars, vols. 1-6 (Krakow: Press of Pedagogical Univ.)

Kusakin, A. V., Mkrtichian, D. E., \& Gamarova, A. Yu. 2001, Inf. Bull. Variable Stars, No. 5106

Kwee, K. K., \& van Woerden, H. 1956, Bull. of the Astron. Inst. of The Netherlands, 12, 327

Mkrtichian, D. E., Kusakin, A. V., Gamarova, A. Yu., et al. 2002, in Observational aspects of pulsating B \& A stars, ed. C. Sterken, \& D. W. Kurtz, PASPC, 256, 259

Mkrtichian, D. E., Kusakin, A. V., Rodríguez, E., et al. 2003a, A\&A, submitted

Mkrtichian, D. E., Nazarenko, V., Gamarova, A. Yu., et al. 2003b, in Interplay between periodic, cyclic and stochastic variability in selected areas of the H-R diagram, ed. C. Sterken, PASPC, 292, 113

Narusawa, S., Waki, Y., Ioroi, M., \& Takeuti, M. 2002, Inf. Bull. Variable Stars, No. 5279

Ohshima, O., Narusawa, S.-Y., Akazawa, H., et al. 2001, AJ, 122, 418

Qian, S. B., 2002, PASP, 114, 650

Qian, S. B., \& Boonrucksar, S. 2002, New Astron., 7, 435

Rodríguez, E., Claret, A., Sedano, J. L., García, J. M., \& Garrido, R. 1998, A\&A, 340, 196

Rodríguez, E., Lopez-Gonzalez, M. J., \& Lopez de Coca, P. 2000, A\&AS, 144, 469

Rodríguez, E., \& Breger, M. 2001, A\&A, 366, 178

Struve, O. 1945, ApJ, 101, 232

van Hamme, W. 1993, AJ, 106, 2096

Vyas, M. L., \& Abhyankar, K. D. 1989, A\&AS, 81, 67

Wilson, R. E., \& Devinney, E. J. 1971, ApJ, 166, 605

Walter, K. 1980, A\&A, 92, 86 